\title{
A smart three-loop fuel cycle architecture for DEMO
}

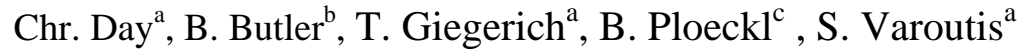 \\ ${ }^{a}$ Karlsruhe Institute of Technology, Karlsruhe (KIT), Germany \\ ${ }^{b}$ Culham Science Centre (CCFE), Abingdon, UK \\ ${ }^{c}$ Max-Planck-Institute of Plasma Physics (IPP), Garching, Germany
}

\begin{abstract}
In the framework of the EUROfusion Programme the EU is preparing the conceptual design of the fuel cycle for a pulsed fusion DEMO. Over the last years, a smart fuel cycle architecture has been developed, driven by the need to reduce the tritium inventory to an absolute minimum. To achieve this goal, batchwise processes used in the fusion fuel cycle so far were replaced by continuous processes wherever possible. This includes the change from discontinuous cryopumping to mercury based continuous vacuum pumping with zero demand on cryoplant power, and the introduction of temperature swing absorption processes for isotope separation in the tritium plant instead of large cryogenic distillation columns with tritiated liquid hold-ups. To circumvent handling of high inventories of tritium-bearing liquid fuel in cryogenic distillation columns of the tritium plant, superpermeable metal foils shall be installed in the divertor ports of the vacuum vessel to extract pure DT continuously from the exhaust gas. Direct internal fuel recycling takes place via the pellet injection system. The paper discusses the design drivers and limitations, and describes the development status of the novel technologies.
\end{abstract}

Keywords: Fuel cycle, tritium, vacuum, pellet, DEMO

\section{Implementation of requirements}

\subsection{Requirements compilation}

Within the framework of the EUROfusion Programme a conceptual design for the fuel cycle of a pulsed fusion DEMO is being prepared. The fuel cycle architecture has been strictly delineated from relevant DEMO high level stakeholder requirements, as listed in the following. The DEMO plant shall [1]:

(i) adopt a tokamak architecture;

(ii) minimise its capital cost (minimise cost of demonstration for taxpayer);

(iii) enable the extrapolation of key performance criteria for a fusion power plant (DEMO must provide a solid basis, also in terms of the technologies involved);

(iv) generate $300-500 \mathrm{MW}$ electrical power to a national grid, continuously for a minimum period of 2 hours;

(v) be designed and have a concept of operation such that a power plant based on it would have a predicted lifetime availability of at least $60 \%$;

(vi) achieve an overall availability of at least 30\%, between commissioning and decommissioning;

(vii) breed tritium exceeding that required for normal operation in order to provide sufficient tritium for the start-up of another plant;

(viii) ensure that it does not exceed its licensed tritium inventory limit (aimed to be at ITER magnitude or less); (ix) provide safe confinement of tritium and radiation (environmental protection and workers' dose minimization).

Here, requirements (ii), (viii) and (ix) represent the main reasons to ask for inventory reduction, whilst the other requirements pose strong limitations on the technology choices. As discussed elsewhere [2, 3], the expected inventories on the basis of an up-scaled ITER fuel cycle are excessive at the level of $10-30 \mathrm{~kg}$ tritium depending on the assumptions taken. This number is found when the batch technology choices for primary pumping (cryopumping), rough pumping (cryopumping and mechanical pumping) and isotope separation (cryogenic distillation) are adopted from ITER. Such a configuration, see Fig. 1, is characterised by a 'oncethrough' architecture in which all tokamak exhaust gas is routed through the tritium plant and finally separated to the level of the pure hydrogen isotopes $\left(\mathrm{H}_{2}, \mathrm{D}_{2}, \mathrm{~T}_{2}\right)$, for re-injection in the torus or for temporary storage. The addition of an outer circuit for tritium breeding at the level needed to achieve tritium self-sufficiency (at a fusion power of $2 \mathrm{GW}$ and a tritium breeding ratio of 1.05) and the technology to recover the tritium from the blanket coolant is adding another contribution to the integral tritium inventory of the DEMO plant.

Such high tritium inventories have an issue in terms of nuclear licensing and from the fact that, within the time scale to start DEMO operation, the available tritium resources may be very limited [4]. The inventory aspect is hence regarded to be a clear showstopper. The novel DEMO fuel cycle architecture is solving this issue. 


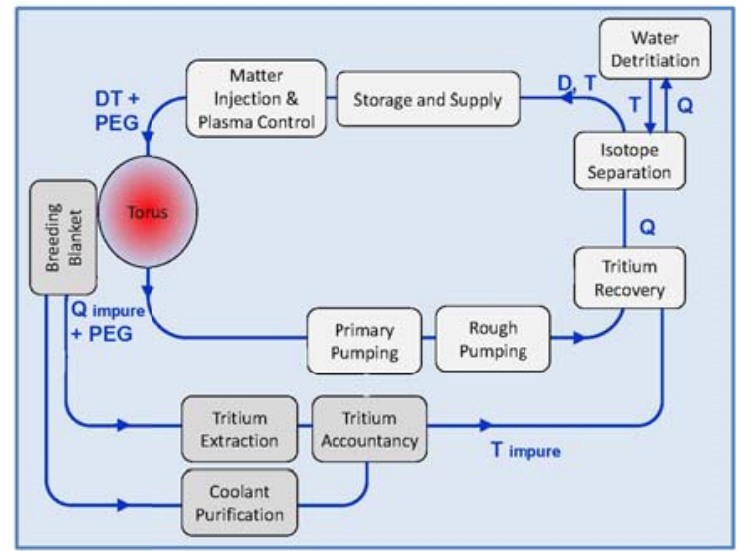

Fig. 1. Conventional once-through architecture of the fusion fuel cycle.

\subsection{Delineation of a smart DEMO fuel cycle architecture}

The fuel cycle of a DEMO/power plant differs from the ITER configuration not only in terms of the blanket systems, but has to meet additional requirements compared to an ITER-class machine, e.g. to ensure a minimum dwell time in between the plasma pulses in order to enable a high availability, and to service a plasma with a range of different plasma enhancement gases for stability and power control at unprecedented amounts at the same time not expecting significantly higher burn-up fractions than anticipated for ITER. In this system engineering exercise, it was also considered that, different to an experimental device such as ITER, DEMO (as well as a Fusion Power Plant) does not require complete isotope separation but only clean fuel, namely $D_{2}-T_{2}$ in a ratio around $1: 1$. As a result, the concept of Direct Internal Recycling (DIR) was integrated [5], leading to two continuous re-cycle loops in addition to an outer loop with classical isotope separation and tritium plant exhaust detritiation technologies, as shown in Fig. 2.

The suggested architecture introduces a separation function close to the divertor. This DIR shortcut reroutes at short processing time the major part of the tritium in the exhaust gas, so that only a minor fraction will have to be routed through the tritium plant, resulting in reduced plant size under nominal operation conditions. This fraction again is distributed in a faster loop with protium removal and isotope re-balancing which produces DT in the wanted composition, and an outermost loop for the tritium recovery duties of the remaining gas [6]. The latter loop also includes the detritiation of tritiated water. As outlined in [7], from the view of the tritium plant, the introduction of the DIR loop with a separation fraction of $80 \%$ - this is the performance which we currently envisage to be feasible would leave a load on the DEMO tritium plant which is by factor 4 lower than in a once-through architecture. The introduction of DIR decouples the tritium plant operation from the tokamak operation [7].

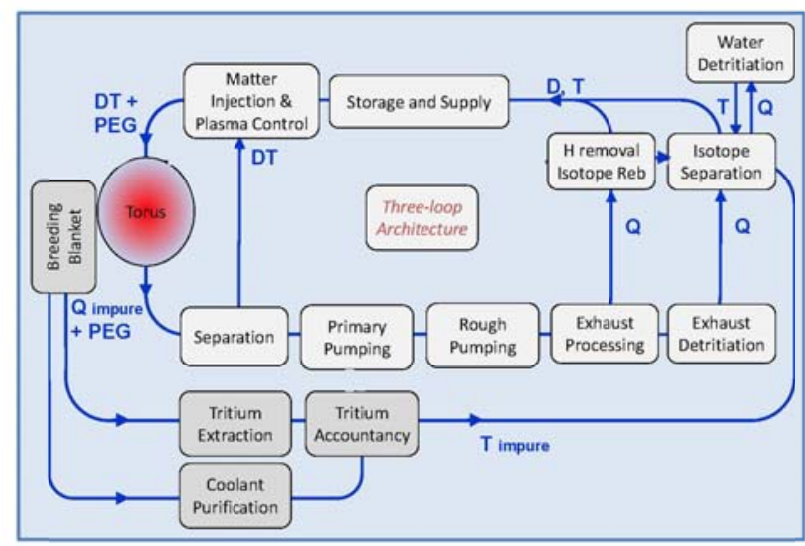

Fig. 2. Reference DEMO fuel cycle architecture with three loops [6, 7].

On top of the improvements given by the smart architecture itself, we plan to replace batchwise processes by continuous processes wherever possible, avoiding accumulation of tritium in each process stage, and immediate reuse of tritium released from tritium breeder blankets. This includes the change from discontinuous torus cryopumping to mercury based continuous vacuum pumping with zero demand on cryoplant power, and the introduction of temperature swing absorption processes for isotope separation in the tritium plant instead of large cryogenic distillation columns with tritiated liquid hold-ups. Superpermeable metal foils are introduced in the divertor ports to separate the DT stream for direct internal recycling to feed the pellet injection systems. The following describes in more detail the technology choices and report the current development status. Implementing the KALPUREX $^{\circledR}$ process [8], Metal Foil Pumps (MFPs) and Linear Diffusion Pumps (LDPs) are used for primary pumping: The MFPs provide a gas separation and split the reactor exhaust gas into an unburnt fuel gas flow (i.e. pure hydrogen gas) and a residual gas flow (hydrogen plus helium and impurities). Bioth flows are being further compressed and pumped by LDPs. This separation is the core function to enable the DIR concept.

Obviously, the fuel cycle scales with the gas throughput, which depends strongly on the plasma scenario and the capability of the divertor to exhaust the particles. As for DEMO both the plasma scenario and the divertor solution are still evolving, we have developed a three-step workflow that allows to extract the technical pumping requirement from the physics of the chosen plasma configuration. This is illustrated in Fig. 3. In the first step, the machine gas throughput is derived from the plasma scenario. Then, the density distribution in the sub-divertor (region below the x-point) is calculated for this gas throughput on the basis of a plasma edge simulation. Finally, the resulting pressure on the pumping slot is extracted. By this scheme, one can immediately study what the impact of a new plasma configuration or changed divertor design on required divertor pumping speed is. This workflow will be exemplified in the following sections. 


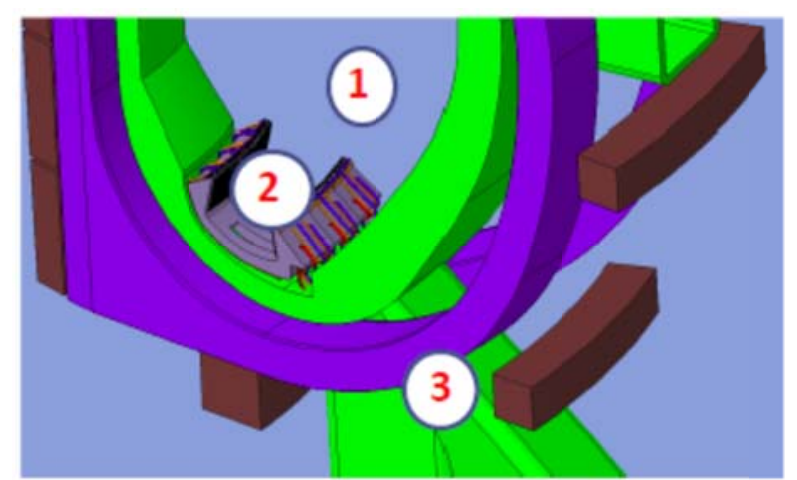

Fig. 3. Illustration of the particle exhaust workflow.

\subsection{Definition of the machine gas throughput}

The starting point to come up with a number for the exhaust gas flow to be taken by the fuel cycle is the nominal fusion power of $2 \mathrm{GW}$, resulting from the high level requirement (iv) above. For steady state DEMO operation, fuel replenishment due to DT burn is rather small, it translates into $7 \cdot 10^{20}$ reactions/s, which corresponds to $2.6 \mathrm{~Pa} \bullet \mathrm{m}^{3} / \mathrm{s}$ formed He. Similarly, the contribution of neutral beam injection is negligible: The injected particle flux translates into $50 \mathrm{MW} / 1 \mathrm{MeV}=0.6$ $\mathrm{Pa} \cdot \mathrm{m}^{3} / \mathrm{s}$; the backflow from the torus into the open NBI duct is of the order of few $\mathrm{Pa} \cdot \mathrm{m}^{3} / \mathrm{s}$. The main contribution to the machine gas throughput comes from fuelling to keep the core density constant, for which we estimate the core part and the scrape-off layer (SOL) separately. The theoretical core fuelling rate has to cover the fuel that is burnt and the additional fuel that is constantly lost from the core due to the density gradient over the separatrix. It therefore depends directly on the transport, and this unknown property is what gives it a high uncertainty. Lang and Fable [9] make an estimate how the theoretical core fuelling rate varies (based on a fit of a set of ASTRA simulations) with the pedestal diffusivity to vary between 0.01 and $0.1 \mathrm{~m}^{2} / \mathrm{s}$, which can be regarded as boundary limit cases. Whereas [10] applies a concept of effective confinement times for which, however, also the unknown particle confinement time can only be estimated. As consequence, the core fuelling is a number with currently very high uncertainty; as average value, we assume $200 \mathrm{~Pa} \bullet \mathrm{m}^{3} / \mathrm{s}$. This flowrate also ensures that the maximum $\mathrm{He}$ concentration limit in the core of 7\% [11] is maintained.

It has been found that the only technology to provide the core fuelling is pellet injection (as is on ITER). However, different from ITER, the target zone is clearly located on the high field side only [12]. This asks for injection velocities around $1 \mathrm{~km} / \mathrm{s}$ via guiding tubes from the high field side (HFS) [9], or with higher velocities in direct line of sight using the vertical DEMO port [13]. The pellets that are injected into the plasma chamber will also lose material on their way through SOL and will contribute to the SOL flow due to deposition and ablation effects of the pellets. The taken loss factor is based on experience. As average we assume a SOL flow of $100 \mathrm{~Pa} \cdot \mathrm{m}^{3} / \mathrm{s}$. On top, one has to add the lost particles in the process of pellet generation, which also have to be pumped by the torus exhaust pumping system. According to an efficiency estimate for HFS pellet injection by Ploeckl [14], we find further $56 \mathrm{~Pa} \bullet \mathrm{m}^{3} / \mathrm{s}$ and, thus, end up with an expected overall pellet efficiency of 200/356=56\%.

It is not sure yet if DT pellet pacing is used for mitigation of ELMs. And even more, there are discussions to change from $\mathrm{H}$-mode to an ELM-free plasma mode. But for the purpose of the present paper, we include this additional flow rate based on the considerations explained in [10].

Radiation control at DEMO will be managed by injection of impurities (plasma enhancement gases, PEG), eventually separately for core and divertor, eventually using different PEG species [15]. The impurity mix must ensure that the power to both divertor targets is below the tolerable threshold, and that the loss power across the separatrix is higher than the $\mathrm{LH}$ threshold power to ensure $\mathrm{H}$ mode being achieved. The concentrations of PEG in the core are expected to be small (some $0.01 \% \mathrm{Xe}$ ) so that this contribution is negligible in terms of the flow rate. It must also be mentioned that a certain PEG concentration in the core is associated with the same or a higher one in SOL, which we do also neglect. Detachment is being achieved using a mixture of Ar and Xe impurities, where Xe is intended to radiate from the core and Ar to further reduce the temperature in the divertor to trigger detachment, to avoid divertor heat overload and excessive surface erosion rates. Divertor gas puffing will be required to reach sufficient neutral pressure in the divertor to further ensure detachment and to reduce power loading of the targets to acceptable values. This additional gas is needed to make sure that the injected radiative seeding gas stays in the divertor region and is not transported out. The required flow rate results from an estimate of friction vs thermal forces for ion retention in the divertor, we use $50 \mathrm{~Pa} \cdot \mathrm{m}^{3} / \mathrm{s}$ in our balance. This gas must not necessarily be fuel gas, it can also be pure deuterium, which may be beneficial for inventory reasons but would potentially add a stronger challenge on exhaust gas treatment. Although the PEG loads are small in terms of concentration, they have a strong impact on the tritium plant, because they require a functionality there to be separated out and recycled.

Gas puffing to reconstitute energy confinement for a metal wall environment reflects the findings with metal wall experiments that additional PEG has to be puffed to achieve stable plasma. Nitrogen and Krypton seeding has been found to work effectivelly. In terms of electrons/s, the seeding rate is roughly of the same order as the core fuelling rate. Due to the potential formation of ammonia, nitrogen has special consequences on the design of the tritium plant, hence we take this contribution on board even if not confirmed yet.

In summary, it is obvious that the throughput to be pumped at DEMO cannot be fixed accurately at the moment. Table 1 summarizes the individual contributions, summing up to $448 \mathrm{~Pa} \cdot \mathrm{m}^{3} / \mathrm{s}$. The values taken represent a conservative case, so that we expect the 
throughput number will become smaller in the future (this has already been the case when compared to previous estimates [16, 17]). On the other side, the numbers have been derived on the basis of an $\mathrm{H}$-mode. If other plasma modes will be used, and/or other than the classical divertor will be used, the throughput number has to be completely revisited.

It must be noted that the density control via pellet injection provides the largest contribution to the machine gas throughput, but also to its uncertainty.

Table 1. Contributions to the machine gas throughput.

\begin{tabular}{ccc}
\hline Purpose & Species & $\begin{array}{c}\text { Quantity } \\
{\left[\mathrm{Pa} \mathrm{m} \mathrm{m}^{3} \mathrm{~s}\right]}\end{array}$ \\
\hline Maintain core density & DT & $\begin{array}{c}200 \\
(22-260)\end{array}$ \\
Fuel losses over SOL & DT & 100 \\
$(22-110)$ \\
Pellet generation losses & DT & 56 \\
ELM pellet pacing & DT & 20 \\
Control of radiative divertor & DT / $\mathrm{D}_{2}$ & 50 \\
seeding and sustainment of \\
detachment & & \\
Confinement recovery of metal & $\mathrm{N}_{2}$ & 20 \\
walls & $\mathrm{Xe}$ & $0.04 \%$ \\
Radiative core seeding & $\mathrm{Xe}+\mathrm{Ar}$ & $0.1 \%$ \\
Radiative Divertor Seeding & & \\
\hline & &
\end{tabular}

\subsection{DEMO divertor neutral pressure}

The throughput and divertor neutral pressure directly point to the required total pumping speed, distributed among a certain number of identical pumps located in divertor pumping ports. We derive the information on divertor neutral pressure from the calculated plasma boundary for a given divertor geometry and the throughput as of Table 1 by means of the DIVGAS code, a Direct Simulation Monte Carlo solver of the Boltzmann equation [18, 19]. Fig. 4 illustrates how the computational domain is defined from the divertor design with variable dome height. The $\mathrm{X}$ and $\mathrm{Y}$ axis denote the global coordinate system of the DEMO machine. In the following example, the dome position has been studied.
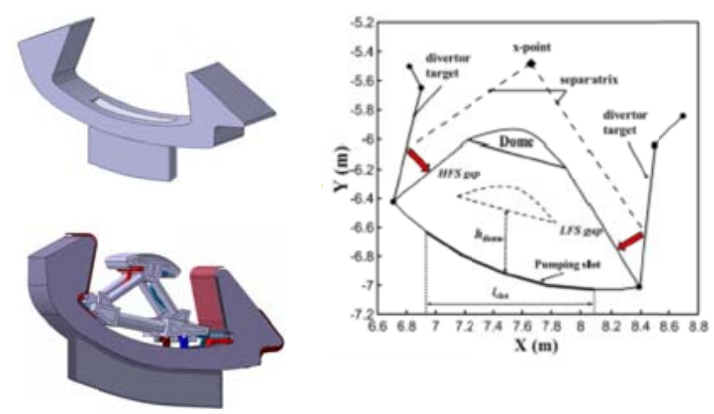

Fig. 4. Divertor designs (left) and corresponding computational domain to derive information on divertor pressure.
It is very important to note that the divertor pressure may be non-uniform. And it is the pressure at the pumped contour only that defines the required pumping speed. This is exemplified in Fig. 5, which illustrates the pressure map for three different configurations of the classical divertor. For this calculation, the poloidal length of the pumping slot is assumed to be $l_{\text {port }}=1.2 \mathrm{~m}$, and the capture coefficient there is taken as $10 \%$, while two dome positions are considlered, namely $\mathrm{h}_{\text {dome }}=0.85 \mathrm{~m}$ and $0.65 \mathrm{~m}$ respectively. The gas enters the sub-divertor area through the two high field side (HFS) and low field side (LFS) gaps with given pressure and temperature reference values equal to $10 \mathrm{~Pa}$ and $4023 \mathrm{~K}$ (or 0.346 $\mathrm{eV}$ ), respectively, whereas the wall temperature is assumed to be $420 \mathrm{~K}$.
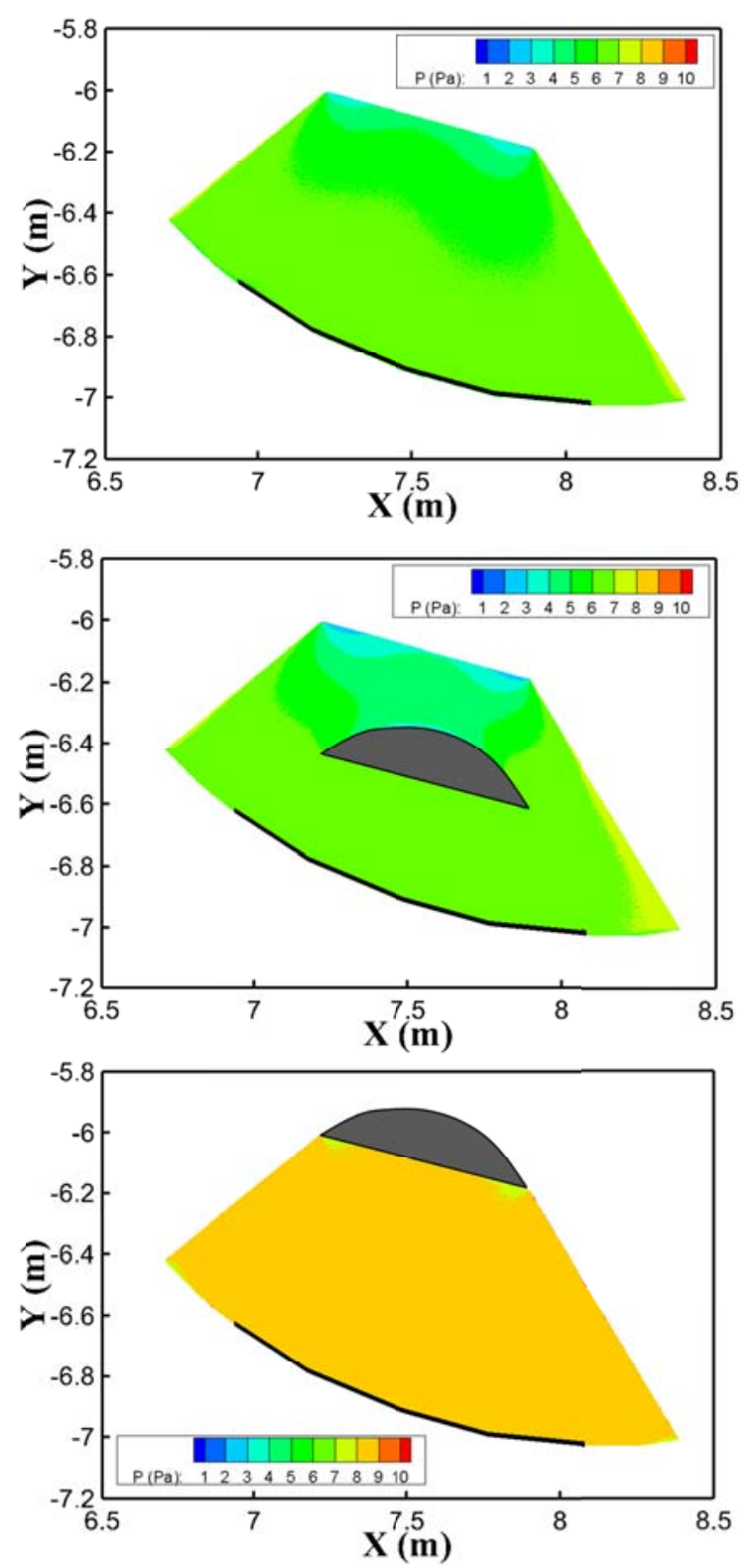

Fig. 5. Divertor total pressure plots calculated for the classical divertor case without (top), with low (middle) and high (bottom) dome position [20].

It is clearly shown how the dome influence the divertor pressure, and hence the pumped flux at given 
connected pumping speed. In this example, the average pressures at the pumped contour were found to lie between $6 \mathrm{~Pa}$ (no dome) and $8 \mathrm{~Pa}$ (high dome). The pumping systems will finally be designed with surplus pumping speed so that the variation of the pumping speed can be used to tune the divertor pressures, to act as an instrument for plasma detachment control and, if needed, to increase the steady-state neutral pressures. This puts an important requirement on the technology choice for the first pump stage.

\section{Technology choices and development programme}

Superpermeable metal foils are introduced in the divertor ports to continuously separate a pure DT stream. As high vacuum pumps, mercury diffusion pumps are under development, and continuously working liquid ring pumps with mercury as operating liquid are employed for rough pumping. In the tritium plant, the inner loop has the function of isotope rebalancing and protium removal to provide DT fuel ready for service. This will be based on temperature swing absorption, which is a semi-continuous technology.

\subsection{Metal foil pumping}

The metal foil pump is planned to be installed between the main torus pumping system and the torus itself. It is based on the principle of superpermeation, which selectively works for hydrogen. Although there was quite some work done on the physics of superpermeation over the last decades, this phenomenon has not yet found its way in a real technical application. Some pump arrangements of metal foils have been suggested [21], but they do not meet the requirements of DEMO, so that the metal foil pump for DEMO still has a very low technical readiness. The European Fusion Programme has identified this as one of 8 key design integration issues of DEMO and has defined a R\&D plan to validate the metal foil pump concept by 2020, with support of experts from Bonch- Bruyevich St Petersburg State University in Russia.

Superpermeation only occurs in surface-limited systems, where the surface energy barrier is only permeable for energetic particles. This introduces the separation effect if there is a way to energize the hydrogenic species of the incoming particle flux, e.g. by applying a (cold) plasma forming hydrogen radicals or ions. All other steps in superpermeation are the same as in classical permeation. Superpermeation allows permeation likelihoods of more than $10 \%$ for incident energetic hydrogen. This is orders of magnitudes higher than in any diffusion-limited system.

A new experimental set-up has just been commissioned at KIT to characterize a plasma source with a tubular metal foil test arrangement, see Figs. 6 and 7. The experimental objective of this facility is to develop a plasma source that is able to cover the full operational pressure range in DEMO (for both burn and dwell phase). According to the current state-of-the-art this would require 3 different technologies, the hot filament for lowest pressures, a microwave plasma for the intermediate range, and a collisional plasma for the highest pressures. First tests with the facility shown in Fig. 6 point to achievable permeation fluxes in the order of $1\left(\mathrm{~Pa} \bullet \mathrm{m}^{3}\right) /\left(\mathrm{s} \bullet \mathrm{m}^{2}\right)$. In parallel to this programme, the effect of the outer magnetic field on the plasma source will be investigated.

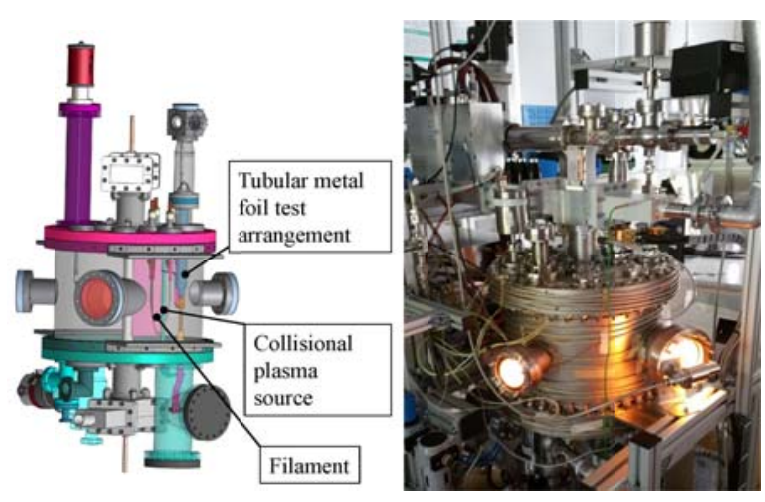

Fig. 6. The new metal foil test facility HERMESplus.

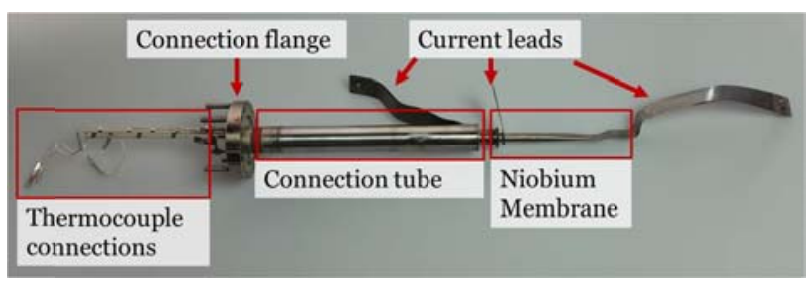

Fig. 7. The tubular metal foil test arrangement (Niobium, $0.1 \mathrm{~mm}$ thick).

\subsection{Novel technology for vacuum pumping}

The major compression of the exhaust gas (in both partial streams, the permeate towards the DIR loop, and the retentate towards the tritium plant) is being performed by a mercury diffusion pump and a mercury based liquid ring pump as roughing pump.

The mercury diffusion pump technology is a very old technology [22], which, however has been replaced in industrial applications in favour of oil diffusion pumps (which are not tritium-compatible and are therefore not an option for the fuel cycle). From the former times, there is distinct experience available for using successfully mercury pumps for tritium pumping [23]. For DEMO, we have modified the classical design of a mercury diffusion pump to have a linear connection flange and a linear arrangement of the nozzles, see Fig. 8 [8]. This design is most versatile and allows for good upscaling which is needed in view of the evolving DEMO design. On top of the inlet cross-section, a twostage baffle system will be installed to liquefy out any backflowing mercury vapour [8]. In case of a safety event resulting in pressures above normal operation, there is an additional closure baffle installed (fast shutter). The current R\&D programme foresees to manufacture such a pump - especially modified with 
additional sensors - and characterize its performance both in terms of pumping speed and in terms of mercury migration control as part of the conceptual design phase of DEMO. Fig. 8 illustrates the design of the test pump, and how such a pump would be integrated with a metal foil pump (eight tubular arrangements (diameter 500 $\mathrm{mm}$, Length $2 \mathrm{~m}$ ) integrated in one module) in a pump cask in one of the DEMO ports [24]. To achieve the high throughputs that typically appear in fusion, the diffusion pump has to be equipped with an additional ejector stage at the outlet.

From the calculated pressures at the pump contour (see 1.4 above), one can derive a required effective pumping speed of the order of $70 \mathrm{~m}^{3} / \mathrm{s}$. Together with the assumed DIR recycling ratio of $80 \%$, this translates in about 10 needed pump ducts (between 9 and 13, if the associated uncertainties are taken into account). More detailed modeling is under way to further reduce the uncertainty of this number.
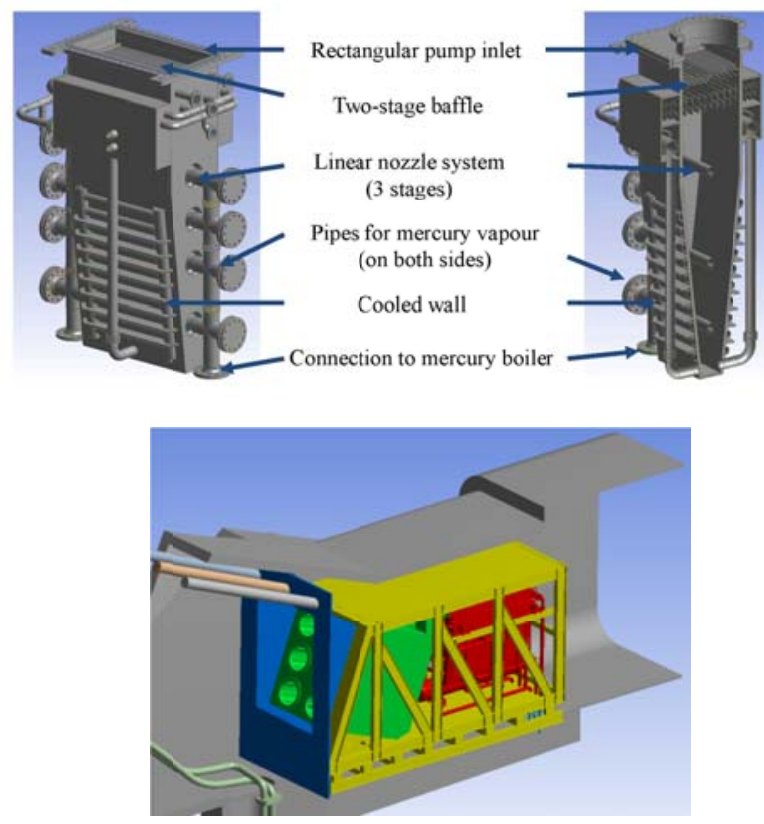

Fig. 8. Top: Current design of a linear diffusion pump (rated $20 \mathrm{~m}^{3} / \mathrm{s}$ ). Bottom: Integration of the diffusion pump (red) with a metal foil pump (green) on a common frame in the lower port.

With this configuration of MFP and LDP, the time needed to pump-down in the dwell phase in between two plasma shots can be estimated. It mainly depends on the outgassing from the vessel walls, and on the required end pressure. Here, DEMO has decided to rely on discharge recovery by EC-assisted start-up, which requires a dwell pressure of only $2 \mathrm{mPa}$ instead of $0.5 \mathrm{mPa}$, as previously $[25,26]$, which allows for reasonable dwell times. The outgassing was assessed with the TESSIM code [27]. With this, the combined outgassing flux (induced thermally and from neutron irradiation) can be pumped out in less than 1000s. Fig. 9 shows the pump-down curves for two different vessel wall cooling scenarios, and for the no-outgassing case for comparison.

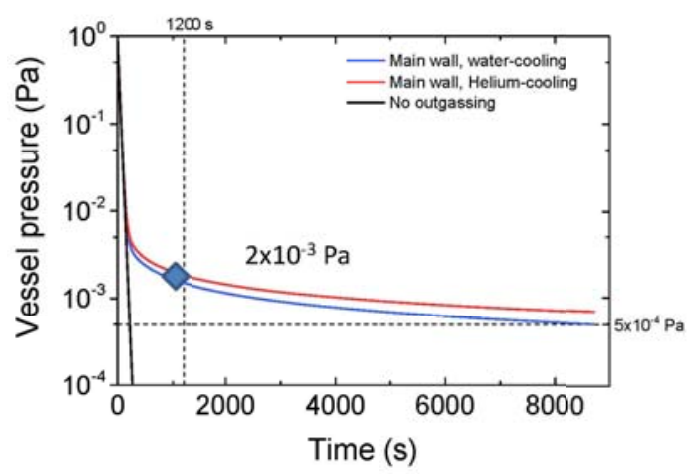

Fig. 9. Dwell pump-down.

As backing pump, DEMO foresees a mercury liquid ring pump which has never been built before. The liquid ring pump technology makes this pump extremely robust, but mercury adds additional requirements on mechanical strength and limits the allowed window of rotor frequency. Within the EUROfusion programme, and together with industry support, already the 2nd generation of such pumps has been reached. They are used - together with diffusion type booster pumps - in a dedicated mechanical pump train to be used in the JET DT campaign [28, 29]. Fig. 10 shows a picture of the pump stand which is currently undergoing acceptance tests at KIT.

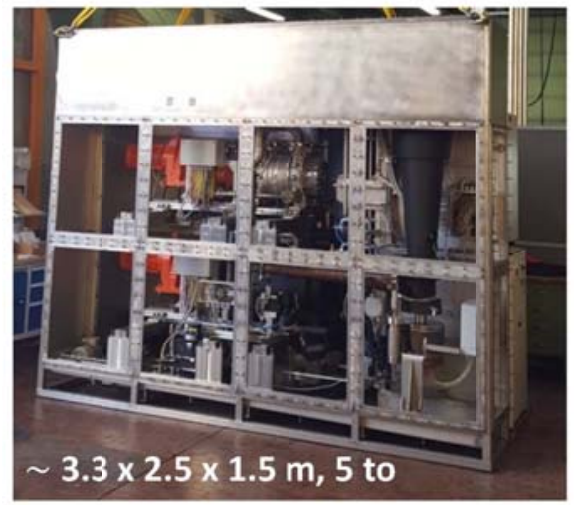

Fig. 10. Mercury based vacuum pump train for JET (during transportation to the commissioning facility).

\subsection{Novel technology for isotope separation}

The smart architecture features two loops inside the tritium plant, the classical outer loop with cryogenic distillation, together with a faster inner one [6]. Both will contain system blocks serving an isotope separation function: The first, isotope rebalancing and protium removal (IR\&PR), sits in the inner loop of the tritium plant and adjusts the composition of the torus exhaust to return a 50:50 mixture of deuterium and tritium, with protium from outgassing being removed. The second, in the outer loop, performs trace tritium recovery from the hydrogen streams produced by the breeder blankets, coolant purification system, exhaust detritiation system and the protium-rich stream from IR\&PR. It is aimed to make the trace tritium recovery isotope separation system to be the only source of hydrogen emissions from 
the tritium plant. The reference technology chosen for the IR\&PR function is a (semi-continuous) thermal cycling temperature swing absorption process, derived from the known TCAP technology [30] to be able to achieve higher efficiencies and thus to minimize the size of the absorption part which is known to scale less good. As alternative approach, a pressure swing adsorption process is under closer investigation within the Prospective R\&D Programme of EUROfusion.

\section{Viability}

An initial viability analysis has been made in order to confirm that the functional and technology choices of the new fuel cycle architecture hold for the DEMO scale. As regards the core fuelling pellet injection systems, frequencies of around $25 \mathrm{~Hz}$ are envisaged. The divertor ports needed for pumping do not conflict with the maintenance systems needed there. It is currently under discussion if the two functions can be integrated on different ports rather than to share on the same, to consider that the requirements of the in-vessel maintenance are different from the maintenance needs of the pump systems. If the advanced fuel cycle is realized, the tritium plant for DEMO will potentially not be larger than that for ITER (see also section 1.2 above). In case of a water-cooled blanket, the coolant purification may be done in an off-site extra facility. A first estimate shows that the global inventory is of the order of $1 \mathrm{~kg}$ (excluding the stored amounts in the U-beds), with less than $100 \mathrm{~g}$ in the blankets, several $100 \mathrm{~g}$ in the tritium plant, less than $100 \mathrm{~g}$ in the fuel injection and vacuum systems, and several $100 \mathrm{~g}$ in the first wall (to be reconfirmed by on-going $R \& D$ programmes with irradiated tungsten materials with tritium exposure).

\section{Conclusions}

The DEMO fuel cycle architecture differs from the classical once-through concept. It features the direct internal recycling loop and two loops inside the tritium plant. We call this new architecture smart, because

(i) it allows to drastically reduce the tritium inventories requiring processing in the Tritium Plant

(ii) it reduces cost (CapEX, OpEx) as cryogenic needs are reduced (completely eliminated for the pumping systems)

(iii) it is derived from the plasma scenario directly.

(iv) it decouples the fuel cycle operation from the plasma physics performance.

(v) it allows to harvest optimization potentials by having the tritium, matter injection and vacuum systems all in one project, and unifies fuel injection and vacuum systems.

\section{Acknowledgments}

This work has been carried out within the framework of the EUROfusion Consortium and has received funding from the EURATOM research and training programme 2014-2018 and 2019-2020 under grant agreement No
633053. The views and opinions expressed herein do not necessarily reflect those of the European Commission.

This work has been done under the Tritium-Matter Injection-Vacuum Programme of the EUROfusion DEMO Project.

The support of A. Livshits and A. Busniuk (BonchBruyevich St Petersburg State University, Russia) in providing the metal foil module and consulting on HERMESplus is gratefully acknowledged. The support of Klaus Schmid (Max-Planck-Institute of Plasma Physics, Garching) for the outgassing calculations is also gratefully acknowledged.

\section{References}

[1] G. Federici et al., Fus. Eng. Des. 136 (2018) 729.

[2] Chr. Day and T. Giegerich, Fus. Eng. Des. 88 (2013) 616.

[3] M. Abdou et al., Fus. Eng. Des. 100 (2015) 2.

[4] M. Kovari et al., Nucl. Fusion 58 (2018) 026010.

[5] Chr. Day et al., Fus. Eng. Des. 109-111 (2016) 299.

[6] R. Lawless et al., Fus. Sci. Technol. 71 (2018) 679.

[7] B. Peters et al., Fus. Eng. Des. 136 (2018) 1467.

[8] T. Giegerich and Chr. Day, Fus. Eng. Des. 89 (2014) 1476.

[9] P. Lang et al., Fus. Eng. Des. 96-97 (2015) 123.

[10] Y. Igitkhanov et al., Fus. Sci. Technol. 72 (2017) 780.

[11] R. Wenninger et al., Nucl. Fusion 57 (2017) 016011.

[12] B. Pégourié et al., $43^{\text {rd }}$ EPS Conf. Plasma Physics, July 2016, Leuven, Belgium, available online: ocs.ciemat.es/EPS2016PAP/pdf/P4.076.pdf

[13] A. Frattolillo et al., Addressing the feasibility of inboard direct-line injection of high speed pellets, for core fueling of DEMO, This Conference.

[14] B. Ploeckl et al., Fus. Eng. Des. 123 (2017) 186.

[15] M. Siccinio et al., Development of a plasma scenario fort he EU-DEMO: Current activities and perspectives, IAEA Fusion Energy Conference, Oct. 2018, Ghandinagar, India.

[16] Chr. Day and T. Giegerich, IEEE Trans. Plasma Science 42 (2014) 1058.

[17] Chr. Day et al., Fus. Eng. Des. 89 (2014) 1505.

[18] S. Varoutis et al., Fus. Eng. Des. 21 (2017) 13.

[19] S. Varoutis et al., Fus. Eng. Des. (2018), accepted for publication.

[20] S. Varoutis et al., Sub-divertor neutral gas dynamics: Integration between the vacuum system and the divertor operation, Symp. On Fusion Engineering (SOFE), June 2017, Shanghai, China.

[21] A. Livshits et al., J. Nucl. Mat. 241-43 (1997) 1203.

[22] W. Gaede, Ann. d. Phys. 46 (1915) 357.

[23] B.D. Abel, J. Vac. Sci. Technol. 15 (1978) 726.

[24] T. Giegerich et al., Preliminary configuration of the torus vacuum pumping system installed in the DEMO lower port, This Conference.

[25] K. Battes and Chr. Day, Fus. Eng. Des. 100 (2015) 431. 
[26] F. Maviglia et al., Overview of DEMO Technology and Scenario Design activities in Europe, $2^{\text {nd }}$ Asia-Pacific Conf. Plasma Physics, Nov. 2018, Kanazawa, Japan.

[27] K. Schmid et al., Phys. Scr. T170 (2017) 014037.

[28] T. Giegerich et al., Fus. Sci. Technol. 68 (2015) 630.

[29] A. Murari et al., Preparing for the scientific and technological exploitation of JET next Tritium campaigns, This Conference.

[30] L.K. Heung et al., Fus. Sci. Technol. 56 (2009) 1471. 